University of South Carolina

Scholar Commons

1986

\title{
Parallel-Plate Electrochemical Reactor Model: A Method for Determining the Time-Dependent Behavior and the Effects of Axial Diffusion and Axial Migration
}

\author{
T. V. Nguyen \\ Texas A \& M University - College Station \\ C. W. Walton \\ Texas A \& M University - College Station \\ Ralph E. White \\ University of South Carolina - Columbia, white@cec.sc.edu \\ J. Van Zee \\ University of South Carolina - Columbia, vanzee@engr.sc.edu
}

Follow this and additional works at: https://scholarcommons.sc.edu/eche_facpub

Part of the Chemical Engineering Commons

\section{Publication Info}

Journal of the Electrochemical Society, 1986, pages 81-87.

(c) The Electrochemical Society, Inc. 1986. All rights reserved. Except as provided under U.S. copyright law, this work may not be reproduced, resold, distributed, or modified without the express permission of The Electrochemical Society (ECS). The archival version of this work was published in Journal of the

Electrochemical Society.

http://www.electrochem.org/

Publisher's Version: http://dx.doi.org/10.1149/1.2108550

DOI: $10.1149 / 1.2108550$

This Article is brought to you by the Chemical Engineering, Department of at Scholar Commons. It has been accepted for inclusion in Faculty Publications by an authorized administrator of Scholar Commons. For more information, please contact digres@mailbox.sc.edu. 
39, 127 (1961).

6. D-T. Chin and C-H. Tsang, This Journal, 125, 1461 (1978).

7. D. R. Gabe and F. C. Walsh, J. Appl. Electrochem., 13, 3 (1983).
8. R. C. Alkire and T-J. Chen, This Journal, 129, 2424 (1982).

9. V.V.Rao and O. Trass, Can.J.Chem. Eng.,42,95 (1964).

10. V. E. Nakoryakov, B. G. Pokusaev, and E. N. Troyan, Int. J. Heat Mass Transfer, 21, 1175 (1978).

\title{
Parallel-Plate Electrochemical Reactor Model
}

\section{A Method for Determining the Time-Dependent Behavior and the Effects of Axial Diffusion and Axial Migration}

\author{
T. V. Nguyen, ${ }^{*}$ C. W. Walton, ${ }^{*}$ and R. E. White** \\ Department of Chemical Engineering, Texas A\&M University, College Station, Texas 77843
}

\author{
J. Van Zee ** \\ Department of Chemical Engineering, University of South Carolina, Columbia, South Carolina 29208
}

\section{ABSTRACT}

A method is presented for determining the effects of time dependence, axial diffusion, and axial migration in a parallel-plate electrochemical reactor (PPER). The method consists of formulating the governing equations and applying a numerical integration technique to solve a set of time-dependent, nonlinear, coupled, multidimensional equations. This formulation reveals that the steady-state performance of the PPER depends on the cell potential and three dimensionless groups. Predictions of the concentration, potential, and local current distributions in a PPER are presented for the electrowinning of copper from an aqueous, hydrochloric acid solution. These predictions show that axial diffusion and axial migration are significant when the aspect ratio (i.e., the ratio of electrode separation to electrode length) is greater than 0.5 .

White et al. (1) presented a model of a parallel-plate electrochemical reactor (see Fig. 1) that has electrodes that are close together. In that model, the effects of axial diffusion and axial migration were unimportant because the distance between the electrodes was assumed to be much smaller than the length of the electrodes. Their model consists of coupled, nonlinear partial differential equations (PDE's) which can be integrated numerically by using a combination of implicit stepping in the axial direction (2) and Newman's technique $(3,4)$ in the normal direction.

If the electrodes are not close together, the material balance equation consists of a set of coupled, nonlinear PDE's that contain second-order derivatives in both the normal and axial directions and cannot, therefore, be solved using the same technique as before (1). The problem can, however, be solved by using a relatively simple, direct numerical integration technique (5) that combines the implicit alternating direction (IAD) algorithm (2, 6-10) with Newman's technique. This IAD-Newman technique can be used to follow the concentration change in a cylindrical catalyst pellet for a nonisothermal, irreversible series reaction (5) and can be applied to electrochemical systems, as discussed here. The IAD-Newman technique is shown to have the advantage of predicting the timedependent behavior as well as the steady-state behavior of a PPER. Also, the effects of axial diffusion and axial migration are assessed by comparing the model predictions of conversion per pass, and concentration, potential, and current distributions for small and large aspect ratios (i.e., $\alpha=S / L$ on the order of 1 ) in a PPER used for the electrowinning of copper from an aqueous, hydrochloric acid solution.

\section{Model Equations}

The dimensionless model equations for a PPER are shown in Table I, where $\eta$ and $\zeta$ are the dimensionless normal and axial directions, respectively, and subscript $j$ is the number corresponding to a particular electrode reaction. The material balance equation for species $\mathbf{i}$ is shown as Eq. [I-1]. The governing equation for the poten-

\footnotetext{
*Electrochemical Society Student Member.
}

**Electrochemical Society Active Member. tial is the electroneutrality equation as shown in dimensionless form in Eq. [I-2]. Eq. [I-3]-[I-6] are the same equations used at the anode and cathode in Ref. (1). These equations are written, in general, for multiple electrode reactions (11), but they do not contain time-dependent derivatives.

In contrast to the boundary conditions at the elec. trodes, the boundary conditions in the axial direction (Eq. [I-7] and [I-9]) include time-dependent partial derivatives. This is because the conditions at the inlet $(\zeta=0)$ and outlet $(\zeta=1)$ of the PPER are formulated to include the fact

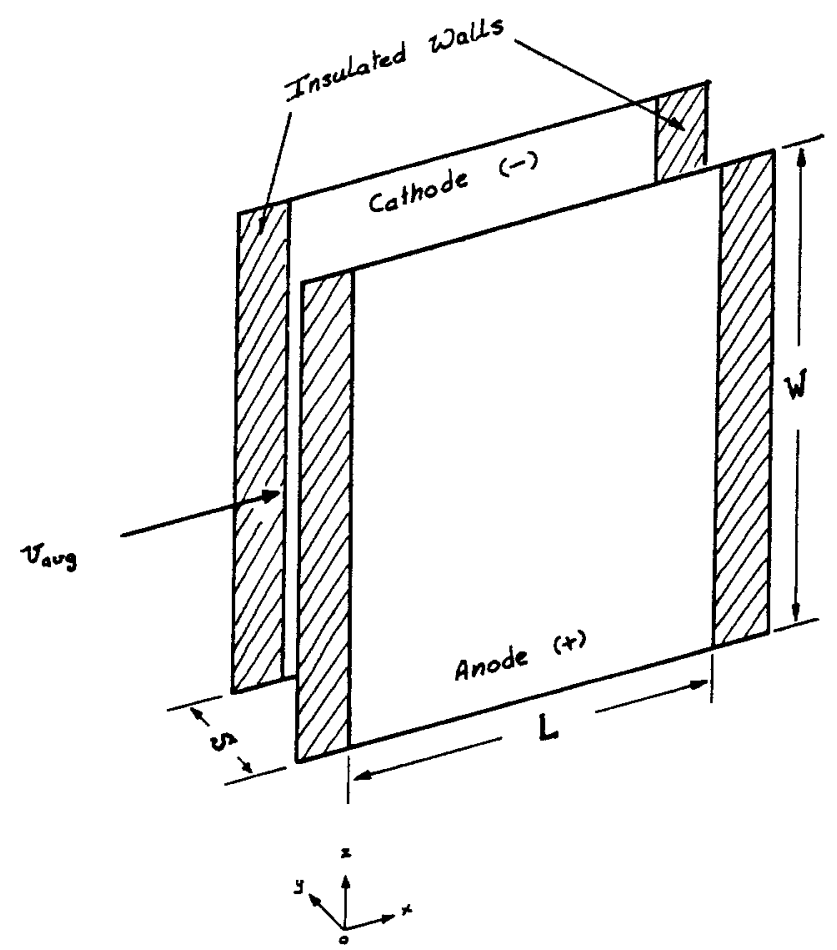

Fig. 1. A schematic of a parallel plate electrochemical reactor (PPER). 
Table I. Dimensionless governing equotions, boundary, and initial conditions for the time-dependent PPER model

For $0<\eta<1$ and $0<\zeta<1$ and $t>0$ (governing equations)

$$
\begin{aligned}
& \frac{S^{2}}{D_{\mathrm{i}}} \frac{\partial \theta_{\mathrm{i}}}{\partial t}=-3 \operatorname{Pe} \alpha \frac{D_{\mathrm{R}}}{D_{\mathrm{i}}}\left(\eta-\eta^{2}\right) \frac{\partial \theta_{\mathrm{i}}}{\partial \zeta}+\alpha^{2} \frac{\partial^{2} \theta_{\mathrm{i}}}{\partial \zeta^{2}}+\frac{\partial^{2} \theta_{\mathrm{i}}}{\partial \eta^{2}} \\
& +\frac{z_{1} \mathbf{F}}{R T}\left[\alpha^{2}\left(\theta_{1} \frac{\partial^{2} \Phi}{\partial \zeta}+\frac{\partial \theta_{1}}{\partial \zeta} \frac{\partial \Phi}{\partial \zeta}\right)+\theta_{1} \frac{\partial^{2} \Phi}{\partial \eta^{2}}+\frac{\partial \theta_{1}}{\partial \eta} \frac{\partial \Phi}{\partial \eta}\right] \\
& \sum_{i} z_{i} c_{i, \text { ref }} \theta_{i}=0 \\
& \text { For } \eta=0 \text { and } 0 \leq \zeta \leq 1 \text { (anode) and } t>0 \\
& \sum_{j} \frac{s_{\mathrm{i}} S \mathrm{i}_{o, \text { ref }}}{D_{\mathrm{i}} c_{\mathrm{i}, \mathrm{ref}} n_{j} \mathbf{F}}\left\{\prod_{\mathrm{i}}\left(\theta_{\mathrm{i}}\right)^{p_{\mathrm{i} j}} \exp \left[\frac{\alpha_{\mathrm{a} j} \mathbf{F}}{R T}\left(V_{\mathrm{a}}-\Phi_{\mathrm{oa}}-U_{j, \mathrm{ref}}\right)\right]\right. \\
& \left.-\prod_{\mathrm{i}}\left(\theta_{\mathrm{i}}\right)^{\boldsymbol{\alpha i} \mathrm{e}} \exp \left[\frac{-\alpha_{\mathrm{c},} \mathbf{F}}{R T}\left(V_{\mathrm{a}}-\Phi_{\mathrm{Da}}-U_{\partial, \mathrm{ref}}\right)\right]\right\}=\frac{\partial \theta_{\mathrm{i}}}{\partial \eta}+\frac{z_{\mathrm{j}} \mathbf{F}}{R T} \theta_{\mathrm{i}} \frac{\partial \Phi}{\partial \eta} \\
& \sum_{i} z_{\mathrm{i}} c_{i, \text { ref }} \theta_{\mathrm{i}}=0
\end{aligned}
$$

For $\eta=1$ and $0 \leq \zeta \leq 1$ (cathode) and $t>0$

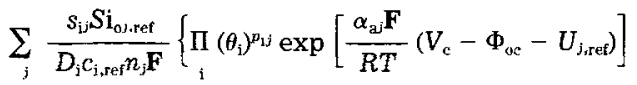

$$
\begin{aligned}
& \left.-\prod_{1}\left(\theta_{\mathrm{i}}\right)^{a_{i j}} \exp \left[\frac{-\alpha_{\mathrm{c}} \mathbf{F}}{R T}\left(V_{\mathrm{c}}-\Phi_{\mathrm{oc}}-U_{j, \mathrm{ref}}\right)\right]\right\}=-\frac{\partial \theta_{\mathrm{i}}}{\partial \eta}-\frac{z_{\mathrm{i}} \mathbf{F}}{R T} \theta_{\mathrm{i}} \frac{\partial \Phi}{\partial \eta} \\
& \sum_{i} z_{i} c_{i, r e f} \theta_{i}=0
\end{aligned}
$$$$
\text { For } \zeta=0 \text { and } 0<\eta<1 \text { (inlet) and } t>0
$$$$
\frac{S^{2}}{D_{1}} \frac{\partial \theta_{i}}{\partial t}=-3 \operatorname{Pe} \alpha \frac{D_{\mathrm{R}}}{D_{\mathrm{i}}}\left(\eta-\eta^{2}\right) \frac{\left(\theta_{\mathrm{i}}-\theta_{\mathrm{i}, \text { feed }}\right)}{\Delta \zeta}+\frac{\alpha^{2}}{\Delta \zeta} \frac{\partial \theta_{\mathrm{i}}}{\partial \zeta}
$$$$
+\frac{\partial^{2} \theta_{1}}{\partial \eta^{2}}+\frac{z_{i} \mathbf{F}}{R T}\left[\frac{\alpha^{2}}{\Delta \zeta} \theta_{\mathrm{i}} \frac{\partial \Phi}{\partial \zeta}+\theta_{\mathrm{i}} \frac{\partial^{2} \Phi}{\partial \eta^{2}}+\frac{\partial \theta_{\mathrm{i}}}{\partial \eta} \frac{\partial \Phi}{\partial \eta}\right]
$$$$
\sum z_{\mathrm{i}} c_{\mathrm{i} . \mathrm{ref}} \theta_{\mathrm{i}}=0
$$

For $\zeta=1$ and $0<\eta<1$ (outlet) and $t>0$

$$
\begin{gathered}
\frac{S^{2}}{D_{\mathrm{i}}} \frac{\partial \theta_{\mathrm{i}}}{\partial t}=-\frac{\alpha^{2}}{\Delta \zeta} \frac{\partial \theta_{\mathrm{i}}}{\partial \zeta}+\frac{\partial^{2} \theta_{\mathrm{i}}}{\partial \eta^{2}}+ \\
\frac{z_{\mathrm{i}} \mathbf{F}}{R T}\left[\frac{-\alpha^{2}}{\Delta \zeta} \theta_{\mathrm{i}} \frac{\partial \Phi}{\partial \zeta}+\theta_{\mathrm{i}} \frac{\partial^{2} \Phi}{\partial \eta^{2}}+\frac{\partial \theta_{\mathrm{i}}}{\partial \eta} \frac{\partial \Phi}{\partial \eta}\right] \\
\sum_{\mathrm{i}} z_{\mathrm{i}} c_{\mathrm{i} . \mathrm{ref}} \theta_{\mathrm{i}}=0
\end{gathered}
$$

For $0 \leq \eta \leq 1$ and $0 \leq \xi \leq 1$ for $t \leq 0$ (initial conditions)

$$
\theta_{1}=1\left(\text { with } \Sigma \zeta_{1} c_{1, \mathrm{ref}} \theta_{1}=0\right.
$$

$\Phi$ no initial condition is needed

$$
\text { (set arbitrarily equal to zero) }
$$

that the electrode reactions occur at $\eta=0$ and $\eta=1$. Equation [I-7] can be obtained by writing the following dimensional material balance equation

$$
\left.\frac{\partial c_{\mathrm{i}}}{\partial t}\right|_{x=0}=-\left.\frac{\partial N_{y \mathrm{i}}}{\partial y}\right|_{x=0}-\left.\frac{\partial N_{x \mathrm{i}}}{\partial x}\right|_{x=0}
$$

and approximating the term $\partial N_{x i} / \partial x$ evaluated at $x=0$ as follows

$$
\left.\frac{\partial N_{x i \mathrm{i}}}{\partial x}\right|_{x=0}=\frac{1}{\Delta x}\left(N_{x \mathrm{i} \mid x=0}-\mathrm{N}_{x \mathrm{i} \mid x=-\Delta x}\right)
$$

Since the flux of each species $i$ prior to entering the reactor $\left(\left.N_{x 1}\right|_{x=-\Delta x}\right)$ is equal to $v_{x} c_{1, \text { feed }}$ (assuming no migration or diffusion in the insulated entrance region), Eq. [2] can be written as

$$
\left.\frac{\partial N_{x \mathrm{i}}}{\partial x}\right|_{x=0}=\frac{1}{\Delta x}\left(\left.N_{x \mathrm{i}}\right|_{x=0}-v_{x} c_{\mathrm{i}, \text { feed }}\right)
$$

Substituting Eq. [3] into Eq. [1] yields a time-dependent, two-dimensional boundary condition for the inlet of the reactor

$$
\left.\frac{\partial c_{\mathrm{i}}}{\partial t}\right|_{x=0}=-\left.\frac{\partial N_{y \mathrm{i}}}{\partial y}\right|_{x=0}-\frac{1}{\Delta x}\left(\left.N_{x \mathrm{i}}\right|_{x=0}-v_{x} c_{\mathrm{i}, \text { feed }}\right)
$$

where $N_{x_{\mathrm{i}}}$ and $N_{y_{\mathrm{i}}}$ are the $x$-component and $y$-component of the flux equation, respectively

$$
\begin{gathered}
N_{x_{\mathrm{i}}}=-D_{\mathrm{i}} \frac{\partial c_{\mathrm{i}}}{\partial x}-\frac{z_{\mathrm{i}} \mathbf{F}}{R T} D_{\mathrm{i}} c_{\mathrm{i}} \frac{\partial \Phi}{\partial x}+v_{x} c_{\mathrm{i}} \\
N_{v_{\mathrm{i}}}=-D_{\mathrm{i}} \frac{\partial c_{\mathrm{i}}}{\partial y}-\frac{z_{\mathrm{i}} \mathbf{F}}{R T} D_{\mathrm{i}} c_{\mathrm{i}} \frac{\partial \Phi}{\partial y}
\end{gathered}
$$

This boundary condition accounts for mass transfer in the axial direction across the entrance boundary $(x=0)$, as well as mass transfer in the normal direction $(y)$ from one electrode to another. Substituting Eq. [5] and [6] into Eq. [4] and putting the resultant equation in dimensionless form yields

$$
\begin{gathered}
\frac{S^{2}}{D_{\mathrm{i}}} \frac{\partial \theta_{\mathrm{i}}}{\partial t}=-3 \operatorname{Pe} \alpha \frac{D_{\mathrm{R}}}{D_{\mathrm{i}}}\left(\eta-\eta^{2}\right) \frac{\left(\theta_{\mathrm{i}}-\theta_{\mathrm{i}, \text { feed }}\right)}{\Delta \zeta}+\frac{\alpha^{2}}{\Delta \zeta} \frac{\partial \theta_{\mathrm{i}}}{\partial \zeta} \\
+\frac{\partial^{2} \theta_{\mathrm{i}}}{\partial \eta^{2}}+\frac{z_{\mathrm{f}} \mathbf{F}}{R T}\left[\frac{\alpha^{2}}{\Delta \zeta} \theta_{1} \frac{\partial \Phi}{\partial \zeta}+\theta_{1} \frac{\partial^{2} \boldsymbol{\Phi}}{\partial \eta^{2}}+\frac{\partial \theta_{\mathrm{i}}}{\partial \eta} \frac{\partial \Phi}{\partial \eta}\right]
\end{gathered}
$$

where $\theta_{i, \text { feed }}$ is the concentration of species $i$ in the feed stream and is equal to one when the feed concentrations are the same as the reference or initial concentrations, as is the case here. Consideration of Eq. [7] shows that it reduces to the inlet conditions $\left[\theta_{i}(\zeta=0)=\theta_{i, \text { feed }}=1\right.$, when $\left.c_{\mathrm{i}, \text { feed }}=c_{\mathrm{i}, \text { ref }}\right]$ used by White et al. (1) when $\alpha$ is small, $\partial \theta_{\mathrm{i}} / \partial t$ $=0$, and $N_{y_{\mathrm{i}}}=0$.

A boundary condition for the outlet of the reactor can be derived similarly by starting with a material balance on species i at $x=L$

$$
\left.\frac{\partial \mathrm{c}_{\mathrm{i}}}{\partial t}\right|_{x=L}=-\left.\frac{\partial N_{y \mathrm{i}}}{\partial y}\right|_{x=L}-\left.\frac{\partial N_{x \mathrm{i}}}{\partial x}\right|_{x=L}
$$

Again, the term $\partial N_{x i} / \partial x$ evaluated at $x=L$ may be approximated by

$$
\left.\frac{\partial N_{x 1}}{\partial x}\right|_{x=L}=\frac{1}{\Delta x}\left(\left.N_{x i}\right|_{x=L+\Delta x}-\left.N_{x 1}\right|_{x=L}\right)
$$

where $\left.N_{x 1}\right|_{x=L+\Delta x}$ is equal to $v_{x} c_{1}(x=L, y, t)$. This balance is based on the assumption that there is no axial diffusion and migration involved beyond $x=L$. This condition, which is often referred to as the "closed-end" boundary condition (12), is satisfied if the reactor empties into a well-mixed tank, for example. This being assumed, Eq. [9] can be written as

$$
\left.\frac{\partial N_{x \mathrm{i}}}{\partial x}\right|_{x=L}=\frac{1}{\Delta x}\left(v_{x} c_{\mathrm{i}}(x=L, y, t)-\left.N_{x \mathrm{i}}\right|_{x=L}\right)
$$

Substituting Eq. [10] into Eq. [8] yields a boundary condition for the outlet of the reactor as follows

$$
\begin{aligned}
\left.\frac{\partial c_{\mathrm{i}}}{\partial t}\right|_{x=L}= & -\left.\frac{\partial N_{y \mathrm{i}}}{\partial y}\right|_{x=L} \\
& -\frac{1}{\Delta x}\left(v_{x} c_{\mathrm{i}}(x=L, y, t)-\left.N_{x \mathrm{i}}\right|_{x=L}\right)
\end{aligned}
$$


Equation [11] written in dimensionless form becomes

$$
\begin{aligned}
\frac{S^{2}}{D_{\mathrm{i}}} \frac{\partial \theta_{\mathrm{i}}}{\partial t} & =-\frac{\alpha^{2}}{\Delta \zeta} \frac{\partial \theta_{\mathrm{i}}}{\partial \zeta}+\frac{\partial^{2} \theta_{\mathrm{i}}}{\partial \eta^{2}} \\
& +\frac{z_{\mathrm{i}} \mathbf{F}}{R T}\left[\frac{-\alpha^{2}}{\Delta \zeta} \theta_{1} \frac{\partial \Phi}{\partial \zeta}+\theta_{1} \frac{\partial^{2} \Phi}{\partial \eta^{2}}+\frac{\partial \theta_{\mathrm{i}}}{\partial \eta} \frac{\partial \Phi}{\partial \eta}\right]
\end{aligned}
$$

Additional discussion of the inlet and outlet boundary conditions is given by Nguyen (13).

\section{Solution Technique}

The set of partial differential equations in Table I can be solved using the IAD-Newman technique (5). The implementation of the IAD-Newman technique requires two finite difference equations for each differential equation, which are used in turn over successive half-time steps. During the first half-time step, the first equation is implicit in one direction ( $\zeta$, for example), and during the remaining half-time step the second equation is implicit in the other direction (i.e., $\eta$, the normal direction). Thus, the unsteady-state material balance equation (Eq. [I-1] in Table I) can be written at the first half-time step

$$
\begin{aligned}
& \frac{S^{2}}{D_{\mathrm{i}}} \frac{\left(\theta^{*}{ }_{\mathrm{i}}-\theta_{\mathrm{i}}\right)}{\Delta t / 2}=-3 \operatorname{Pe} \alpha \frac{D_{\mathrm{R}}}{D_{\mathrm{i}}}\left(\eta-\eta^{2}\right) \frac{\partial \theta^{*}}{\partial \zeta}+\alpha^{2} \frac{\partial^{2} \theta^{*}}{\partial \zeta^{2}}+\frac{\partial^{2} \theta_{\mathrm{i}}}{\partial \eta^{2}} \\
& +\frac{z_{\mathrm{i}} \mathbf{F}}{R T}\left[\alpha^{2}\left(\theta^{*}{ }_{\mathrm{i}} \frac{\partial^{2} \Phi^{*}}{\partial \zeta^{2}}+\frac{\partial \theta^{*}}{\partial \zeta} \frac{\partial \Phi^{*}}{\partial \zeta}\right)+\theta_{\mathrm{i}} \frac{\partial^{2} \Phi}{\partial \eta^{2}}+\frac{\partial \theta_{\mathrm{i}}}{\partial \eta} \frac{\partial \Phi}{\partial \eta}\right]
\end{aligned}
$$

and at the second half-time step

$$
\begin{aligned}
& \frac{S^{2}}{D_{\mathrm{i}}} \frac{\left(\theta^{* *}{ }_{\mathrm{i}}-\theta^{*}\right)}{\Delta t / 2}= \\
& \quad-3 \operatorname{Pe} \alpha \frac{D_{\mathrm{R}}}{D_{\mathrm{i}}}\left(\eta-\eta^{2}\right) \frac{\partial \theta^{*}{ }_{\mathrm{i}}}{\partial \zeta}+\alpha^{2} \frac{\partial^{2} \theta^{*}{ }_{\mathrm{i}}}{\partial \zeta^{2}}+\frac{\partial^{2} \theta^{* *} *_{\mathrm{i}}}{\partial \eta^{2}}+\frac{z_{1} \mathbf{F}}{R T} . \\
& {\left[\alpha^{2}\left(\theta^{*}{ }_{i} \frac{\partial^{2} \Phi^{*}}{\partial \zeta^{2}}+\frac{\partial \theta^{*} \mathrm{i}}{\partial \zeta} \frac{\partial \Phi^{*}}{\partial \zeta}\right)+\theta^{* *} \frac{\partial^{2} \Phi^{* *}}{\partial \eta^{2}}+\frac{\partial \theta^{* *}}{\partial \eta} \frac{\partial \Phi^{* *}}{\partial \eta}\right]}
\end{aligned}
$$

where $*$ and $* *$ designate the dependent variables for the first and second half-time step: the lack of a ${ }^{*}$ superscript indicates previous time-step or starting values. The derivatives in Eq. [13] and [14] are expressed in finite difference expressions according to the superscript designation. It is important to point out that, in this work, the concentration of each species $i$ (the term $\theta_{i}$ not part of the derivatives) in Eq. [13] and [14] was treated as unknown at each half-time step [as suggested in Ref. (5)] and then treated as shown in Eq. [13] and [14], and it was found that both approaches yield the same results. However, in the latter approach, convergence can be obtained with fewer iterations.

Table II. Implementation scheme for IAD-Newman solution to PPER equations

First half-time step [implicit in axial ( $\zeta$ direction]

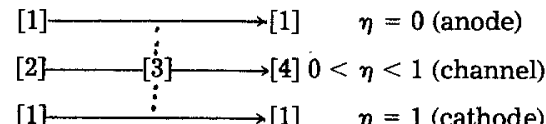

$$
\begin{aligned}
& (\zeta=0) \longrightarrow(\zeta=1) \\
& \theta^{*}{ }_{1}=\theta_{1} \text { and } \Phi^{*}=\Phi
\end{aligned}
$$$$
\frac{S^{2}}{D_{1}} \frac{\left(\theta^{*}{ }_{i}-\theta_{1}\right)}{\Delta t / 2}=-3 \operatorname{Pe} \alpha \frac{D_{\mathrm{R}}}{D_{1}}\left(\eta-\eta^{2}\right) \frac{\left(\theta^{*}{ }_{1}-\theta_{\text {i.feed }}\right)}{\Delta \zeta}+\frac{\alpha^{2}}{\Delta \zeta} \frac{\partial \theta^{*}{ }_{1}}{\partial \zeta}
$$$$
+\frac{\partial^{2} \theta_{1}}{\partial \eta^{2}}+\frac{z_{1} \mathbf{F}}{R T}\left[\frac{\alpha^{2}}{\Delta \zeta} \theta^{*}{ }_{1} \frac{\partial \Phi^{*}}{\partial \zeta}+\theta_{1} \frac{\partial^{2} \Phi}{\partial \eta^{2}}+\frac{\partial \theta_{1}}{\partial \eta} \frac{\partial \Phi}{\partial \eta}\right]
$$

$$
\begin{aligned}
& \frac{S^{2}}{D_{\mathrm{i}}} \frac{\left(\theta^{*}{ }_{1}-\theta_{1}\right)}{\Delta t / 2}=-3 \operatorname{Pe} \alpha \frac{D_{\mathrm{R}}}{D_{\mathrm{i}}}\left(\eta-\eta^{2}\right) \frac{\partial \theta^{*}{ }_{1}}{\partial \zeta}+\alpha^{2} \frac{\partial^{2} \theta^{*}{ }_{\mathrm{i}}}{\partial \zeta^{2}}+\frac{\partial^{2} \theta_{1}}{\partial \eta^{2}} \\
& +\frac{z_{1} \mathbf{F}}{R T}\left[\alpha^{2}\left(\theta^{*}{ }_{1} \frac{\partial^{2} \Phi}{\partial \zeta^{2}}+\frac{\partial \theta_{1}^{*}}{\partial \zeta} \frac{\partial \Phi^{*}}{\partial \zeta}\right)+\theta_{\mathrm{i}} \frac{\partial^{2} \Phi}{\partial \eta^{2}}+\frac{\partial \theta_{1}}{\partial \eta} \frac{\partial \Phi}{\partial \eta}\right] \\
& \sum_{\mathrm{i}} z_{\mathrm{i}} c_{\mathrm{i}, \text { ref }} \theta_{\mathrm{i}}=0 \\
& \frac{S^{2}}{D_{\mathrm{i}}} \frac{\left(\theta^{*}{ }_{1}-\theta_{\mathrm{i}}\right)}{\Delta t / 2}=-\frac{\alpha^{2}}{\Delta \zeta} \frac{\partial \theta^{*}{ }_{\mathrm{i}}}{\partial \zeta}+\frac{\partial^{2} \theta_{\mathrm{i}}}{\partial \eta^{2}}+ \\
& \frac{z_{\mathrm{i}} \mathbf{F}}{R T}\left[\frac{-\alpha^{2}}{\Delta \zeta} \theta^{*} \frac{\partial \Phi^{*}}{\partial \zeta}+\theta_{1} \frac{\partial^{2} \Phi}{\partial \eta^{2}}+\frac{\partial \theta_{\mathrm{i}}}{\partial \eta} \frac{\partial \Phi}{\partial \eta}\right] \\
& \sum_{\mathbf{i}} z_{\mathrm{i}} c_{\mathrm{i}, \text { ref }} \theta^{*}{ }_{\mathrm{i}}=0
\end{aligned}
$$

Second half-time step [implicit in normal ( $\eta$ ) direction]$$
\begin{array}{cccc}
{[5]} & & {[5]} & \\
\downarrow & & \downarrow & \\
{[6]} & \cdots & {[8]} & \cdots \\
\downarrow & & \downarrow & \\
{[7]} & & {[7]} & \\
{[7} & & &
\end{array}
$$$$
\text { [5] } \eta=0 \text { (anode) }
$$$$
[9] 0<\eta<1 \text { (channel) }
$$$$
\text { [7] } \eta=1 \text { (cathode) }
$$$$
(\zeta=0) \longrightarrow(\zeta=1)
$$

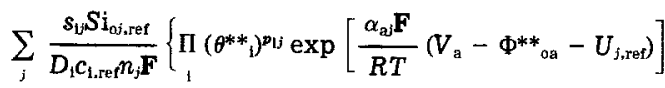$$
\left.-\Pi_{1}\left(\theta^{* *}\right)^{\alpha_{j}} \exp \left[\frac{-\alpha_{\mathrm{cj}} \mathbf{F}}{R T}\left(V_{\mathrm{a}}-\Phi^{* *}{ }_{\mathrm{oa}}-U_{j, \mathrm{ret}}\right)\right]\right\}
$$$$
=\frac{\partial \theta_{1}^{* *}}{\partial \eta}+\frac{z_{1} \mathbf{F}}{R T} \theta_{1}^{* *} \frac{\partial \Phi^{* *}}{\partial \eta}
$$$$
\sum_{i} z_{i} c_{i, \text { ref }} \theta^{* *}{ }_{i}=0
$$$$
\frac{S^{2}}{D_{\mathrm{i}}} \frac{\left(\theta^{* *}{ }_{\mathrm{i}}-\theta^{*}{ }_{\mathrm{i}}\right)}{\Delta t / 2}=-3 \operatorname{Pe} \alpha \frac{D_{\mathrm{R}}}{D_{\mathrm{i}}}\left(\eta-\eta^{2}\right) \frac{\left(\theta^{*}{ }_{\mathrm{i}}-\theta_{\mathrm{i}, \text { feed }}\right)}{\Delta \zeta}+\frac{\alpha^{2}}{\Delta \zeta} \frac{\partial \theta^{*}{ }_{\mathrm{i}}}{\partial \zeta}
$$$$
+\frac{\partial^{2} \theta^{* *}}{\partial \eta_{1}^{2}}+\frac{z_{1} \mathbf{F}}{R T}\left[\frac{\alpha^{2}}{\Delta \zeta} \theta_{1}^{*} \frac{\partial \Phi^{*}}{\partial \zeta}+\theta^{* *}{ }_{1} \frac{\partial^{2} \Phi^{* *}}{\partial \eta^{2}}+\frac{\partial \theta^{* *}}{\partial \eta} \frac{\partial \Phi^{* *}}{\partial \eta}\right]
$$$$
\sum_{i} z_{\mathrm{i}} c_{\mathrm{i}, \mathrm{rer}} \theta^{* *}{ }_{1}=0
$$$$
\sum_{j} \frac{s_{\mathrm{j}} S \mathrm{i}_{\mathrm{i}_{j}, \mathrm{ref}}}{D_{\mathrm{i}} \mathrm{c}_{\mathrm{i}, \mathrm{ref}} n_{j} \mathbf{F}}\left\{\Pi\left(\theta^{* *}\right)^{p_{\mathrm{ij}}} \exp \left[\frac{\alpha_{\mathrm{aj}} \mathbf{F}}{R T}\left(V_{\mathrm{c}}-\Phi^{* *}{ }_{\mathrm{oce}}-U_{j, \mathrm{ree}}\right)\right]\right.
$$$$
\left.-\underset{\mathrm{i}}{-}\left(\theta^{* *_{\mathrm{i}}}\right)^{\alpha_{\mathrm{ij}}} \exp \left[\frac{-\alpha_{\mathrm{c}} \mathbf{F}}{R T}\left(V_{\mathrm{c}}-\Phi^{* *_{\mathrm{oe}}}-U_{j, \mathrm{ret}}\right)\right]\right\}
$$$$
=-\frac{\partial \theta^{* *}{ }_{\mathrm{i}}}{\partial \eta}-\frac{z_{\mathrm{i}} \mathbf{F}}{R T} \theta^{* *}{ }_{\mathrm{i}} \frac{\partial \Phi^{* * *}}{\partial \eta}
$$$$
\sum_{1} z_{1} c_{1, \text { rer }} \theta^{* *}{ }_{1}=0
$$

$\frac{S^{2}}{D_{1}} \frac{\left(\theta^{* *}{ }_{1}-\theta^{*}{ }_{1}\right)}{\Delta t / 2}=-3 \operatorname{Pe} \alpha \frac{D_{\mathrm{R}}}{D_{1}}\left(\eta-\eta^{2}\right) \frac{\partial \theta^{*}{ }_{1}}{\partial \zeta}+\alpha^{2} \frac{\partial^{2} \theta^{*}{ }_{1}}{\partial \zeta^{2}}+\frac{\partial^{2} \theta^{* *}{ }_{1}}{\partial \eta^{2}}$$$
+\frac{z_{\mathbf{i}} \mathbf{F}}{R T}\left[\alpha^{2}\left(\theta^{*}{ }_{1} \frac{\partial^{2} \Phi^{*}}{\partial \zeta^{2}}+\frac{\partial \theta^{*}}{\partial \zeta} \frac{\partial \Phi^{*}}{\partial \zeta}\right)+\theta^{* *}{ }_{1} \frac{\partial^{2} \Phi^{* *}}{\partial \eta^{2}}+\frac{\partial \theta^{* *}{ }_{1}}{\partial \eta} \frac{\partial \Phi^{* *}}{\partial \eta}\right]
$$

$$
\sum_{i} z_{\mathrm{j}} c_{\mathrm{i}, \text { ref }} \theta^{* * *} \mathrm{i}=0
$$

$$
\begin{gathered}
\frac{S^{2}}{D_{1}} \frac{\left(\theta^{* *} *_{i}-\theta^{*}\right)}{\Delta t / 2}=-\frac{\alpha^{2}}{\Delta \zeta} \frac{\partial \theta^{*}}{\partial \zeta}+\frac{\partial^{2} \theta^{* *}{ }_{i}}{\partial \eta^{2}} \\
+\frac{z_{i} \mathbf{F}}{R T}\left[\frac{-\alpha^{2}}{\Delta \zeta} \theta^{*}{ }_{i} \frac{\partial \Phi^{*}}{\partial \zeta}+\theta^{* *}{ }_{1} \frac{\partial^{2} \Phi^{* *}}{\partial \eta^{2}}+\frac{\partial \theta^{* *}}{\partial \eta} \frac{\partial \Phi^{* *}}{\partial \eta}\right] \\
\sum_{\mathrm{i}} z_{\mathrm{i}} \mathrm{c}_{\mathrm{i}, \mathrm{rer}} \theta^{* *{ }_{\mathrm{i}}}=0
\end{gathered}
$$


Table II illustrates the implementation of the IADNewman technique to the complete set of governing equations shown in Table I. Grid Eq. [1] shows that the unknowns along the electrodes are set equal to their values at the beginning of the time step (i.e., $\theta^{*}{ }_{i}=\theta_{i}$ and $\Phi^{*}$ $=\Phi)$. This is done because the boundary conditions for the electrodes contain derivatives in the $\eta$ direction only and are satisfied in the next half-time step (implicit in the $\eta$ direction, grid Eq. [5] and [7]). Similarly, grid Eq. [2], [3], and [4] show that the previous half-time step values of the concentration and potential are used only for the finite difference expressions for the derivatives in the radial direction and for half of the expression in the time derivative. The equations for the second half-time step treat the derivatives in the radial direction implicitly. Note that at the anode and cathode, grid Eq. [5] and [7] do not include any previous values from the intermediate half-time step. On the other hand, the grid Eq. [6] and [9] applied at the inlet and exit of the reactor include intermediate halftime step values in the axial direction derivatives and in the time derivative. Grid Eq. [8] also includes intermediate values for the axial and time-dependent derivatives in the same manner as grid Eq. [3] includes the previous half-time step values.

After formulating the equations as shown in Table II the numerical integration is accomplished by applying Newman's BAND $(J)(14)$ first at the anode (i.e., using the grid Eq. [1]) with $J=1$ and $J=N J$ corresponding to $\zeta=0$ and $\zeta=1$, respectively. Then $\operatorname{BAND}(J)$ is applied successively at each row of mesh points using grid Eq. [2], [3], and [4] up to and including the cathode using grid equation [1] with $J=1$ and $J=N J$ again corresponding to $\zeta=0$ and $\zeta=1$, respectively. Note that each application of $\operatorname{BAND}(J)$ during these first half-time steps is coupled in the radial direction through the previous time-step value and coupled in the axial direction through the dependent values $\theta^{*}$ and $\Phi^{*}$.

For the second half-time step, the numerical integration is accomplished by applying $\operatorname{BAND}(J)$ first at the inlet of the reactor using the grid Eq. [5], [6], and [7] with $J=1$ and $J=N J$ corresponding to $\eta=0$ and $\eta=1$, respectively. Then $\operatorname{BAND}(J)$ is applied successively at each column of mesh points using the grid Eq. [5], [8], and [7] up to and including the exit of the reactor using the grid Eq. [5], [9], and [7] with $J=1$ and $J=N J$ again corresponding to $\eta=0$ and $\eta=1$. The values of the dependent variables $\theta^{* *}$ and $\Phi^{* *}$ are the results at a full time-step $\Delta t$ and they constitute the previous time step values to be used at the next first half-time step.

As is the case for all finite difference techniques, the accuracy of the solution depends on the number of grid points used. For three-digit accuracy [this accuracy was obtained by adding additional mesh points in both the $\eta$ and $\zeta$ directions and decreasing the time step size $(\Delta t)$ until the results no longer changed to within three digits], the central processing unit (CPU) time for the model with the axial diffusion and migration terms included was 70-100 times longer than the time required to solve the model without the axial diffusion and migration terms. The total execution time was approximately $30,000 \mathrm{CPU}$ seconds on a CDC-Cyber 170-825 with the axial diffusion and migration terms included.

\section{Results and Discussion}

The method can be used to solve the model equations for a typical parallel-plate system for the electrowinning of copper from an aqueous hydrochloric acid solution where the reaction at the anode is

$$
\mathrm{CuCl}_{3}{ }^{2-} \rightleftharpoons \mathrm{CuCl}^{+}+2 \mathrm{Cl}^{-}+e^{-} \text {(anode, reaction 1) }
$$

and the reaction at the cathode is

$$
\mathrm{CuCl}_{3}{ }^{2-}+e^{-} \rightleftharpoons \mathrm{Cu}+3 \mathrm{Cl}^{-} \text {(cathode, reaction 2) }
$$

Table III gives the values of all fixed parameters used in the study of the model for these electrode reactions. The
Table III. Values for fixed parameters used in the model

Kinetic and thermodynamic

\begin{tabular}{lcccccl}
\hline $\begin{array}{l}\text { Reaction } \\
(j)\end{array}$ & $\begin{array}{l}10^{4} \mathrm{i}_{\mathrm{o}_{j} \text {, ref }} \\
\left(\mathrm{A} / \mathrm{cm}^{2}\right)^{\mathrm{a}}\end{array}$ & $\alpha_{\mathrm{a} j}$ & $\alpha_{\mathrm{c}^{j}}$ & $n_{j}$ & $\begin{array}{l}U_{j}^{\theta^{\theta}} \\
(\mathrm{V})^{\mathrm{h}}\end{array}$ & $\begin{array}{l}U_{j, \text { ref }} \\
(\mathrm{V})^{\mathrm{c}}\end{array}$ \\
\hline 1 & 1.0 & 0.5 & 0.5 & 1 & 0.438 & 0 \\
2 & 1.0 & 0.5 & 0.5 & 1 & 0.233 & 0.1144
\end{tabular}

\begin{tabular}{|c|c|c|c|c|c|c|}
\hline \multirow{2}{*}{$\begin{array}{l}\text { Component } \\
\text { (i) }\end{array}$} & \multicolumn{3}{|c|}{ Reaction $1(j=1)$} & \multicolumn{3}{|c|}{ Reaction $2(j=2)$} \\
\hline & $s_{\mathrm{i} j}$ & $p_{\mathrm{i} j}$ & $q_{\mathrm{i} j}$ & $s_{\mathrm{i} j}$ & $p_{i j}$ & $q_{1^{j}}$ \\
\hline $\mathrm{H}^{+}$ & 0 & 0 & 0 & 0 & 0 & 0 \\
\hline $\mathrm{Cl}^{-}$ & -2 & 0 & 2 & $\begin{array}{l}0 \\
3\end{array}$ & $\begin{array}{l}0 \\
3\end{array}$ & 0 \\
\hline $\mathrm{CuCl}^{2-\mathrm{d}}$ & 1 & 1 & 0 & -1 & 0 & 1 \\
\hline $\mathrm{CuCl}^{-}$ & -1 & 0 & 1 & 0 & 0 & 0 \\
\hline$V_{\text {cathode }}=0 \mathrm{~V}$ & \multicolumn{3}{|c|}{$S=0.1 \mathrm{~cm}$} & \multicolumn{3}{|c|}{$\mathrm{W}=10 \mathrm{~cm}$} \\
\hline
\end{tabular}

$T=298.15 \mathrm{~K}$

Transport and reference concentrations

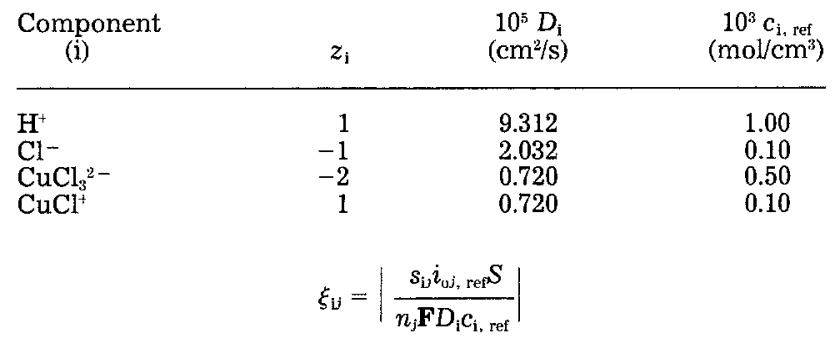

Component (i)

Reaction 1

Reaction 2

\begin{tabular}{lll}
\hline $\mathrm{H}^{+}$ & 0.0000 & 0.0000 \\
$\mathrm{Cl}^{-}$ & 0.1020 & 0.1530 \\
$\mathrm{CuCl}_{3}{ }^{2-}$ & 0.0288 & 0.0288 \\
$\mathrm{CuCl}^{+}$ & 0.1440 & 0.0000 \\
\hline
\end{tabular}

a Chosen arbitrarily.

${ }^{\mathrm{b}}$ See Ref. (11) and (16) of White et al. (1).

c The open-current potential of reaction $j$ at the reference concentrations relative to reaction 1 .

${ }^{\mathrm{d}}$ Designated as the limiting reactant.

effects of axial diffusion and migration on the performance of a parallel-plate electrochemical reactor can be studied in terms of both their influence on conversion per pass and the concentration, potential, and local current density distributions. The steady-state values can be obtained with the time-dependent model by stepping through time until the results no longer change to within three digits. The dynamic behavior of the concentration distribution of the reactant $\mathrm{CuCl}_{3}{ }^{2}$ in the electrochemical reactor as the system approaches steady state is demonstrated in Fig. 2 and 3 for the case of $\alpha=1$. Figure 2 shows the concentration distribution of $\mathrm{CuCl}_{3}{ }^{2}-$ in the reactor at time equal to 2 min and Fig. 3 shows a lower concentration distribution at steady state which occurs in $6.5 \mathrm{~min}$, which is what would be expected during a start-up situation, since $\mathrm{CuCl}_{3}{ }^{2}$ - is consumed at both electrodes. It is interesting to note that, when $\alpha=0.01$, steady state was achieved in only $5 \mathrm{~min}$, which may be an important consideration when testing control schemes with laboratory scale reactors.

Effect of axial diffusion and migration on conversion.-The conversion per pass of a species $\mathrm{i}\left(\mathrm{CPP}_{1}\right)$ can be defined as (1)

$$
\mathrm{CPP}_{\mathrm{i}}=\left|\theta_{\mathrm{i}, \text { eeed }}-\theta_{\mathrm{i}, \text { avg }}(t, \zeta=1)\right|
$$

According to the model equations in Table I, the steadystate value of $\mathrm{CPP}_{\mathrm{i}}$ is a function of four independent variables

$$
\begin{gathered}
E_{\text {cell }}=\left(V_{\mathrm{a}}-V_{\mathrm{c}}\right) \\
\operatorname{Pe} \alpha=\frac{2 S^{2} v_{\text {avg }}}{D_{\mathrm{R}} L}
\end{gathered}
$$




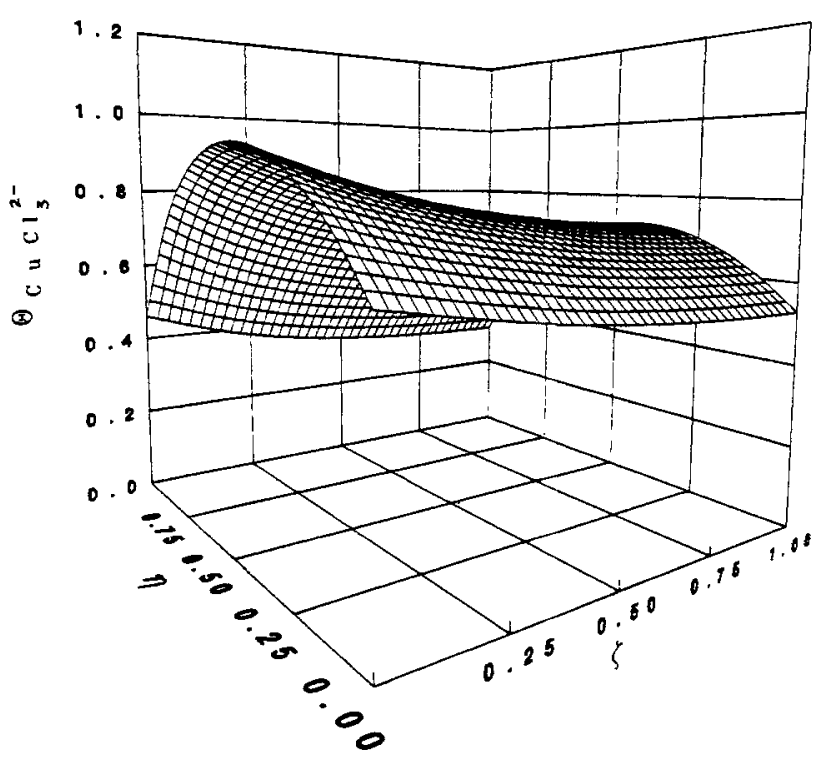

Fig. 2. Concentration distribution of $\mathrm{CuCl}_{3}{ }^{2-}$ in the PPER at 2 min $\left(\alpha=1, \operatorname{Pe} \alpha=10 ; E_{\text {cell }}=0.4 \mathrm{~V}\right)$.

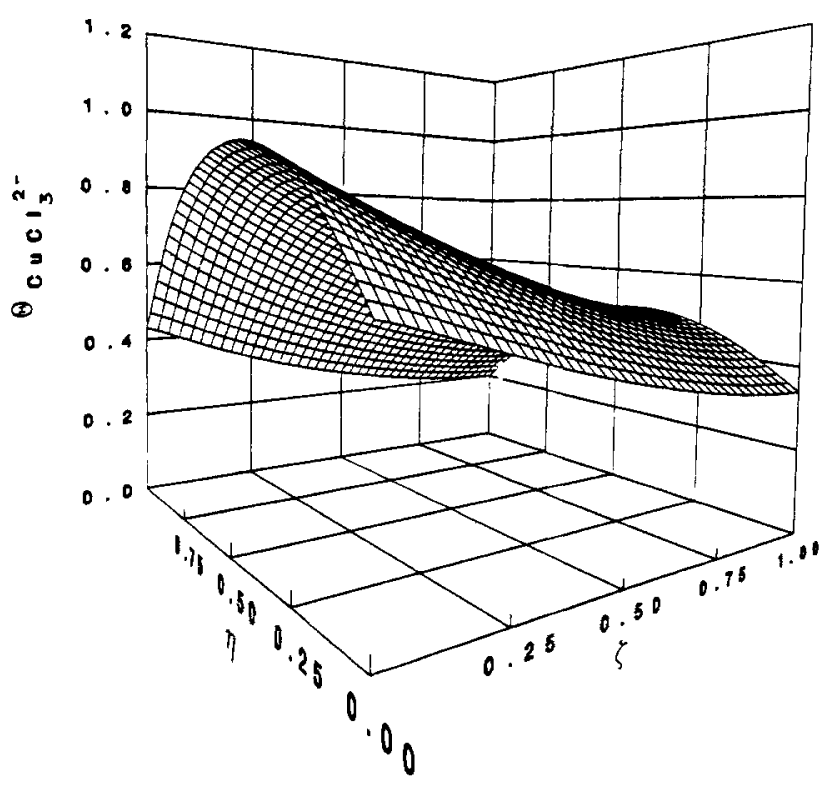

Fig. 3. Concentration distribution of $\mathrm{CuCl}_{3}{ }^{2-}$ in the PPER at steady state $\left(\alpha=1, \operatorname{Pe} \alpha=10 ; \boldsymbol{E}_{\text {cell }}=0.4 \mathrm{~V}\right)$.

$$
\alpha^{2}=\frac{S^{2}}{L^{2}}
$$

and

$$
\xi_{1 j}=\frac{s_{1 j} i_{0 j, \mathrm{re}} S}{n_{j} \mathbf{F} D_{\mathrm{R}} c_{1, \text { ref }}}
$$

Pe $\alpha$ and $\alpha^{2}$ are chosen instead of Pe and $\alpha$ because of the way they appear in the material balance equation (Eq. [I-1] in Table I). While $E_{\text {cell }}$ and the dimensionless groups $\operatorname{Pe} \alpha$ and $\xi_{1 j}$ (see Table III) are always important, the dimensionless variable $\alpha^{2}$ can be neglected when the distance between the electrodes $(S)$ is much smaller than the length $(L)$ of the electrodes, i.e., $S / L<<1$, [as done by White et al. (1)]. That is, the effects of axial diffusion and migration are negligible and can be ignored when the aspect ratio $(\alpha=S / L)$ is small. In a simple model of a parallel-plate cell by Pickett (15), the quantity $\mathrm{CPP}_{\mathrm{i}}$ (Pickett's fractional conversion, $f_{\mathrm{R}}$; his Eq. [5.44]) is defined as

$$
\mathrm{CPP}_{\mathrm{i}}=1-\exp \left\{-2.33\left(\frac{\mathrm{A}}{\operatorname{ReScWS}}\right)^{2 / 3}\right\}
$$

where $\operatorname{Re}=v_{\mathrm{avg}} 2 S / \nu, \mathrm{Sc}=\nu / D_{\mathrm{R}}$, and $A$ are the Reynolds number, the Schmidt number, and the electrode area of the reactor, respectively. Note that Pickett's $B$ is replaced by $W$ to match the nomenclature in this paper. It is important to point out here that Pickett's model was derived for the case of a single-electrode reaction (i.e., only one electrode is considered and at this electrode the reactant is consumed by a single reaction). Therefore, to compare properly Pickett's model with the results obtained with this model, where the reactant is consumed at both electrodes, it is necessary to double the surface area (notice the 2 in front of variable $A$ in Eq. [22])

$$
\mathrm{CPP}_{\mathrm{i}}=1-\exp \left\{-2.33\left(\frac{2 \mathrm{~A}}{\operatorname{ReScWS}}\right)^{2 / 3}\right\}
$$

After substitution of variables, Eq. [22] can be written as

$$
\mathrm{CPP}_{\mathrm{i}}=1-\exp \left\{-2.33\left(\frac{\mathrm{Pe} \alpha}{2}\right)^{-2 / 3}\right\}
$$

This expression for $\mathrm{CPP}_{\mathrm{i}}$ is incomplete because it does not include the correct dependence of $\mathrm{CPP}_{\mathrm{i}}$ on important cell design variables such as the distance between the electrodes $(S)$ and the cell potential $\left(E_{\text {cell }}\right)$. Furthermore, Eq. [23] is restricted to cases of very low conversion per pass because it is derived with the assumption of constant reactant concentration in the bulk solution.

Table IV shows a comparison between three models at various Pe $\alpha$ : Pickett's model (15), the model of White et al. (1), which omits the effects of axial diffusion and axial migration, and this model. It is important to point out that the parallel plate model as presented by White et al. (1) does not include reactions occurring at the entrance points of the electrodes. However, by using the inlet boundary condition (Eq. [7]) derived in this work, with the axial diffusion and migration terms and the timedependent term neglected, the model of White et al. (1) would then include reactions occurring at $\zeta=0$. The values for the model of White et al. (1) as given in Table IV have been corrected to include the entrance points of the electrodes by making an extra step down the reactor, which is equivalent to using the inlet boundary condition (Eq. [9]) directly. Uncorrected values are 1\% lower.

It is possible to vary $\alpha$ while holding Pe $\alpha$ and other independent variables constant, as shown in Table IV, by changing $L$ and $v_{\mathrm{avg}}$. Note that as the aspect ratio $\alpha$ increases, which is equivalent to increasing the effects of axial (back) mixing in the reactor, the predicted $\mathrm{CPP}_{\mathrm{i}}$ for $\mathrm{CuCl}_{3}{ }^{2-}$ decreases. Consequently, the percentage error for neglecting the effects of axial diffusion and migration in predicting the conversion per pass can be as large as $11 \%$

\begin{tabular}{|c|c|c|c|c|c|c|c|}
\hline \multirow[b]{2}{*}{$\operatorname{Pe} \alpha$} & \multicolumn{6}{|c|}{$\mathrm{CPP}$ of $\mathrm{CuCl}_{3}{ }^{2}-\left(E_{\text {cell }}=0.4 \mathrm{~V}\right.$} & \multirow[b]{2}{*}{$\begin{array}{c}\% \\
\text { Error }\end{array}$} \\
\hline & $\begin{array}{c}v_{\text {avg }} \\
(\mathrm{cm} / \mathrm{s})\end{array}$ & $\begin{array}{c}L \\
(\mathrm{~cm})\end{array}$ & $\alpha$ & $\begin{array}{l}\text { Pickett's } \\
\text { Model }\end{array}$ & $\underset{1^{\mathrm{a}}}{\text { Model }}$ & $\begin{array}{c}\text { Model } \\
2^{\mathbf{b}}\end{array}$ & \\
\hline $\begin{array}{l}10 \\
10 \\
10 \\
10 \\
10\end{array}$ & $\begin{array}{l}0.036 \\
0.0036 \\
0.0018 \\
0.00072 \\
0.00036\end{array}$ & $\begin{array}{l}10 \\
1 \\
0.5 \\
0.2 \\
0.1\end{array}$ & $\begin{array}{l}0.01 \\
0.10 \\
0.20 \\
0.50 \\
1.00\end{array}$ & $\begin{array}{l}0.549 \\
0.549 \\
0.549 \\
0.549 \\
0.549\end{array}$ & $\begin{array}{l}0.606 \\
0.606 \\
0.606 \\
0.606 \\
0.606\end{array}$ & $\begin{array}{l}0.606 \\
0.602 \\
0.600 \\
0.585 \\
0.548\end{array}$ & $\begin{array}{r}0.0 \\
0.6 \\
1.0 \\
3.6 \\
10.6\end{array}$ \\
\hline $\begin{array}{l}25 \\
25\end{array}$ & $\begin{array}{l}0.0900 \\
0.0009\end{array}$ & $\begin{array}{c}10 \\
0.1\end{array}$ & $\begin{array}{l}0.01 \\
1.00\end{array}$ & $\begin{array}{l}0.351 \\
0.351\end{array}$ & $\begin{array}{l}0.334 \\
0.334\end{array}$ & $\begin{array}{l}0.334 \\
0.327\end{array}$ & $\begin{array}{l}0.0 \\
2.1\end{array}$ \\
\hline $\begin{array}{l}50 \\
50\end{array}$ & $\begin{array}{l}0.1800 \\
0.0018\end{array}$ & $\begin{array}{l}10 \\
0.1\end{array}$ & $\begin{array}{l}0.01 \\
1.00\end{array}$ & $\begin{array}{l}0.239 \\
0.239\end{array}$ & $\begin{array}{l}0.193 \\
0.193\end{array}$ & $\begin{array}{l}0.193 \\
0.196\end{array}$ & $\begin{array}{l}0.0 \\
1.5\end{array}$ \\
\hline $\begin{array}{l}100 \\
100\end{array}$ & $\begin{array}{l}0.3600 \\
0.0036\end{array}$ & $\begin{array}{c}10 \\
0.1\end{array}$ & $\begin{array}{l}0.01 \\
1.00\end{array}$ & $\begin{array}{l}0.158 \\
0.158\end{array}$ & $\begin{array}{l}0.107 \\
0.107\end{array}$ & $\begin{array}{l}0.107 \\
0.111\end{array}$ & $\begin{array}{l}0.0 \\
3.6\end{array}$ \\
\hline
\end{tabular}
at $\alpha=1$ for the case $\mathrm{Pe} \alpha=10$. However, for aspect ratios less than 0.5 the model of Ref. (1) is accurate to about $5 \%$,

Toble IV. The effect of axial diffusion and migration on the conversion-per-pass of the reactant $\mathrm{CuCl}_{3}{ }^{2-}$

Note: Values given above for models 1 and 2 are accurate to three significant figures.

a The model of White et al. (1).

b Present model.

c $\%$ Error $=100 \cdot \mid$ Model $1-$ Model 2|/Model 2. 


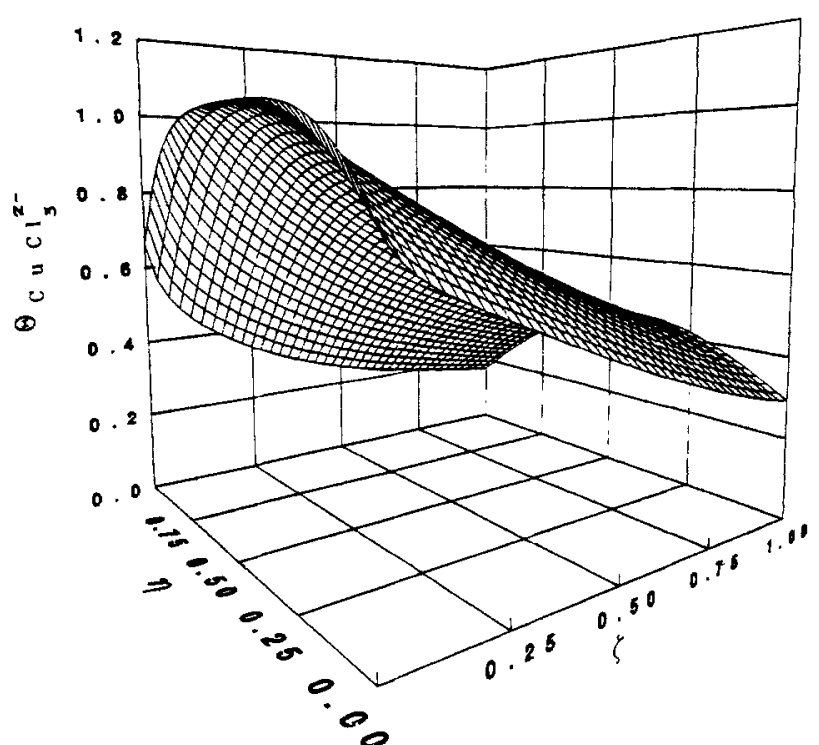

Fig. 4. Steady-state concentration distribution of $\mathrm{CuCl}_{3}{ }^{2-}$ in the PPER when the effects of axial diffusion and migration are not important $\left(\alpha=0.01 ; \operatorname{Pe} \alpha=10 ; E_{\text {cell }}=0.4 \mathrm{~V}\right)$.

and its use is recommended for a considerable saving in computer time. It is coincidental here that the solutions from this work agree to a large extent with the solutions from Pickett's model (15) for the case $\mathrm{Pe} \alpha=10$. The agreement is limited for other cases. Note also that as Peo increases (by increasing the velocity of the electrolyte), the effects of axial diffusion and axial migration become less significant. It is interesting that at higher $\operatorname{Pe} \alpha$ (50 and 100 ) the effect of change $\alpha$ has an opposite effect on the conversion of the reactant, as compared to the cases of $\mathrm{Pe} \alpha=10$ and 25.

Effects of axial diffusion and migration on concentration, potential, and current density distributions.-The effects of axial diffusion and migration on the concentration distribution in the reactor are shown in Fig. 4 and 3 for the cases of $\alpha=0.01$ and $\alpha=1$. These figures show that as the aspect ratio $\alpha$ increases (i.e., as the effects of axial diffusion and axial migration increase) the concentration distribution in the reactor becomes more uniform. This is what one would expect as the effects of axial-diffusion and migration increase and cause the parallelplate reactor to become well mixed. The same effects are observed with the potential distribution in the reactor and the local current density distribution along the surface of the electrodes, as shown in Fig. 5, 6, and 7.

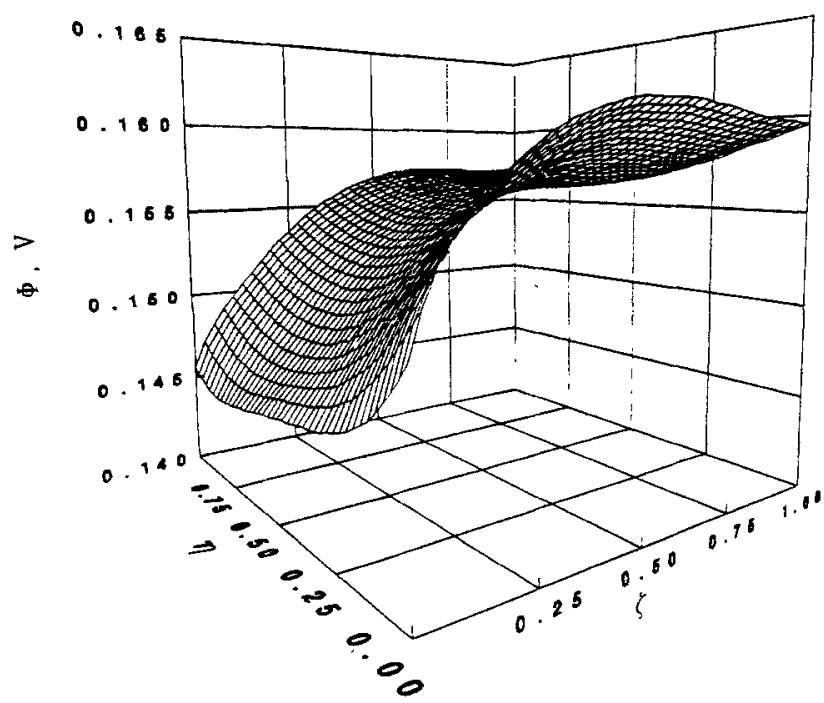

Fig. 5. Steady-state potential distribution in the PPER when the effects of axial diffusion and migration are not important $(\alpha=0.01$; $P e \alpha=10 ; E_{\text {cell }}=0.4 \mathrm{~V}$ ).

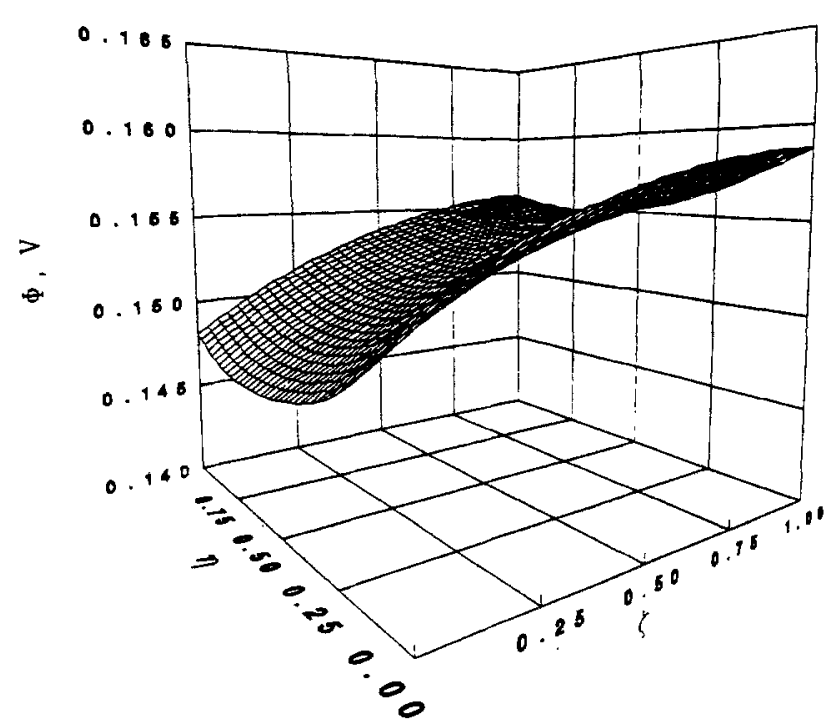

Fig. 6. Steady-state potential distribution in the PPER when the effects of axial diffusion and migration are important $(\alpha=1 ; \mathrm{Pe} \alpha=$ 10; $E_{\text {cell }}=0.4 \mathrm{~V}$ ).

Figure 6, for the case of significant axial diffusion and migration $(\alpha=1)$, shows a more uniform potential distribution and a smaller potential drop from the entrance to the exit of the reactor than that shown in Fig. 5 for $\alpha=$ 0.01 . Figure 7 clearly illustrates the effects of axial diffusion and migration on the distribution of the local current density along the anode. (The current density distribution for the cathode is the same, but opposite in sign.) Note the more uniform current density distribution for $\alpha=1$ (Fig. 7).

The effects of axial (upstream) diffusion and axial migration on the concentration distribution at the entrance of the reactor can be observed by comparing Fig. 4 and 3 . Without the effects of upstream diffusion and migration (that is, when $\alpha=S / L$ is very small) the concentration of each species $i$ in the bulk solution at the entrance of the reactor approaches its feed concentration, as expected. Exceptions to this condition are regions very close to the

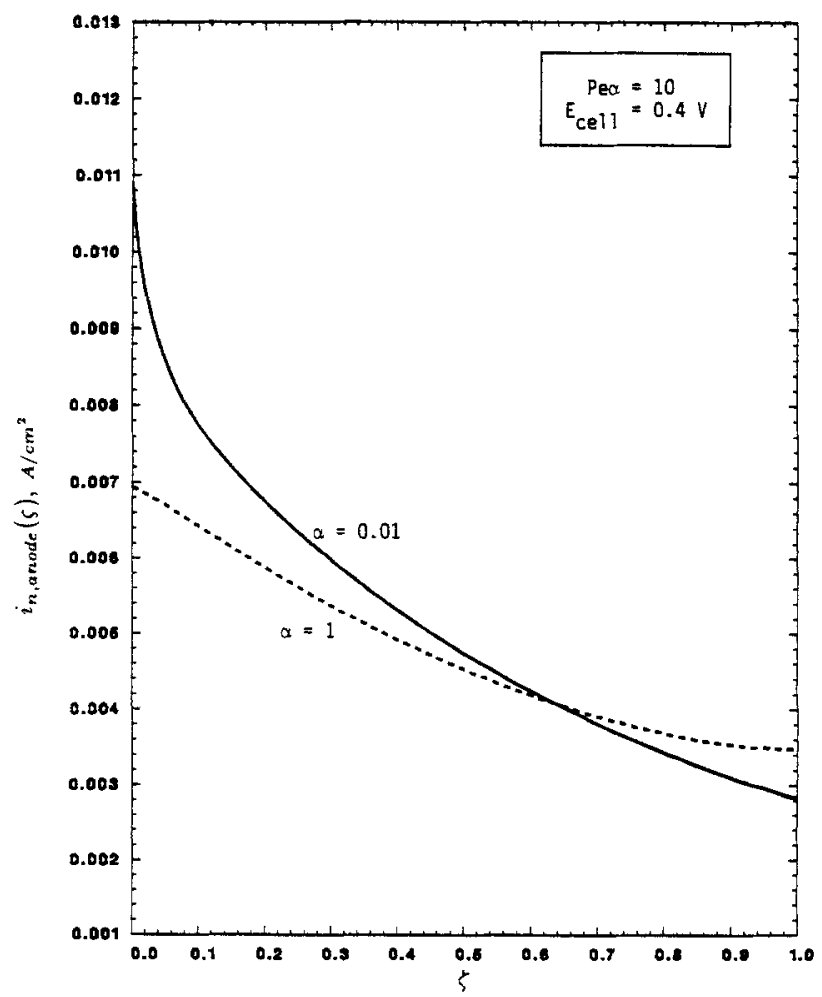

Fig. 7. The effects of axial diffusion and migration on the distribution of current density along the anode. 
surface of the electrodes where there is a large normal $(\eta)$ concentration gradient as the result of reactions occurring at the end points of the electrodes.

\section{Conclusions}

The comparative analysis of the effects of axial diffusion and migration on the parallel-plate reactor shows that when the aspect ratio $\geq 0.5$ the effects of axial diffusion and migration should be included to obtain accurate results. It is found that for an aspect ratio less than 0.5 , these effects may be neglected, which results in considerable savings in computer time. Finally, the timedependent model and the IAD-Newman technique presented here may be a useful design tool for investigating the effects of various design characteristics on the dynamic behavior and the performance of the reactor.

Manuscript submitted June 13, 1985; revised manuscript received Sept. 10, 1985.

\section{LIST OF SYMBOLS}

A electrode area of electrochemical reactor $\left(\mathrm{cm}^{2}\right)$

$c_{\mathrm{i}} \quad$ concentration of species i $\left(\mathrm{mol} / \mathrm{cm}^{3}\right)$

$c_{i, f e d}$ concentration of species $i$ in the feed stream $\left(\mathrm{mol} / \mathrm{cm}^{3}\right)$

$c_{i, 0} \quad$ concentration of species $i$ at the electrode surface $\left(\mathrm{mol} / \mathrm{cm}^{3}\right)$

$c_{1, \text { ref }}$ concentration of species $i$ in the initial feed stream (reference) $\left(\mathrm{mol} / \mathrm{cm}^{3}\right)$

$D_{\mathrm{i}} \quad$ diffusion coefficient of species $\mathrm{i}\left(\mathrm{cm}^{2} / \mathrm{s}\right)$

$D_{\mathrm{R}}$ diffusion coefficient of limiting reactant, $\mathrm{cm}^{2} / \mathrm{s}$

$E_{\text {cell }}$ cell potential $\left(V_{\mathrm{a}}-V_{\mathrm{c}}\right)(\mathrm{V})$

F Faraday's constant (96,487 C/mol of electrons)

$I_{\text {avg }}$ average current density $\left(\mathrm{A} / \mathrm{cm}^{2}\right)$

$i_{n j}$ normal component of current density due to reaction $j\left(\mathrm{~A} / \mathrm{cm}^{2}\right)$

$i_{0 j, \text { ref }}$ exchange current density of reaction $j$ at reference concentrations $\left(\mathrm{A} / \mathrm{cm}^{2}\right)$

$L \quad$ electrode length $(\mathrm{cm})$

$n_{j} \quad$ number of electrons involved in reaction $j$

$N_{i} \quad$ flux vector of species i $\left(\mathrm{mol} / \mathrm{cm}^{2} / \mathrm{s}\right)$

$N_{y_{\mathrm{i}}}$ normal component of the flux ( $y$-direction) of species $\mathrm{i}\left(\mathrm{mol} / \mathrm{cm}^{2} / \mathrm{s}\right)$

$N_{x_{1}} \quad$ flux of species $i$ in the $x$-direction $\left(\mathrm{mol} / \mathrm{cm}^{2} / \mathrm{s}\right)$

$p_{1 j} \quad$ anodic reaction order of species $i$ in reaction $j$

$\mathrm{Pe} \quad$ Peclet number $\left(=2 S v_{\text {ave }} / D_{\mathrm{R}}\right)$

$q_{i j} \quad$ cathodic reaction order of species $i$ in reaction $j$

$R$ gas law constant $(8.314 \mathrm{~J} / \mathrm{mol} / \mathrm{K})$

$s_{\mathrm{ij}} \quad$ stoichiometric coefficient of species $\mathrm{i}$ in reaction $j$

$S$ electrode gap (cm)

$t \quad$ time (s)

$T$ temperature $(\mathrm{K})$

$U_{j \text {,ref }}$ open-circuit potential of reaction $j$ at reference conditions $(V)$

$v_{\text {avg }}$ average velocity of the electrolyte $(\mathrm{cm} / \mathrm{s})$
$V_{\mathrm{a}} \quad$ anode potential (V)

$V_{\mathrm{c}}$ cathode potential $(\mathrm{V})$

W electrode width (cm)

$x$ axial coordinate $(\mathrm{cm})$

$\Delta x \quad$ axial mesh size $(\mathrm{cm})$

$y$ normal coordinate $(\mathrm{cm})$

$z_{i} \quad$ charge number of species $i$

\section{Greek characters}

aspect ratio $S / L$

$\alpha_{\text {aj }}$ anodic transfer coefficient for reaction $j$

$\alpha_{c j} \quad$ cathodic transfer coefficient for reaction $j$

$\zeta$ dimensionless axial coordinate $(x / L)$

$\Delta \zeta \quad$ dimensionless axial mesh size

$\eta$ dimensionless normal coordinate $(y / S)$

$\theta_{\mathrm{i}}$ dimensionless concentration of species $i\left(c_{\mathrm{i}} / c_{\mathrm{i}, \mathrm{ref}}\right)$

$\nu \quad$ kinematic viscosity $\left(\mathrm{cm}^{2} / \mathrm{s}\right)$

$\xi_{\mathrm{i} j} \quad$ dimensionless reaction rate parameter

$\Phi$ solution potential (V)

$\Phi_{\text {oa }}$ solution potential at the anode (V)

$\Phi_{\text {oc }}$ solution potential at the cathode (V)

\section{Subscripts}

i number corresponding to a particular chemical species

$j$ number corresponding to a particular electrode reaction

\section{REFERENCES}

1. R. E. White, M. Bain, and M. Raible, This Journal, 130, 1037 (1983)

2. B. Carnahan, H. A. Luther, and J. O. Wilkes, "Applied Numerical Methods," John Wiley and Sons, New York (1969).

3. J. Newman, Ind. Eng. Chem. Fundam., Y, 514 (1968).

4. R. E. White, ibid., 17, 367 (1978).

5. J. Van Zee, M. A. Edmund, and R. E. White, ibid., 19, $438(1980)$.

6. D. W. Peaceman and H. H. Rachford, J. SIAM, 3, 28 (1955).

7. J. Douglas, Jr., ibid., 3, 42 (1955).

8. P. L. T. Brian, A.I.Ch.E. J., 7, 367 (1961).

9. J. Douglas, Jr., J. SIAM, 4, 41 (1962).

10. J. Douglas, Jr., and J. E. Gunn, Numer. Math., 6, 428 (1964).

11. R. E. White, S. E. Lorimer, and R. Darby, This Journal, 130, 1123 (1983).

12. C. D. Holland and R. G. Anthony, "Fundamentals of Chemical Reaction Engineering," Prentice-Hall, Englewood Cliffs, NJ (1979).

13. T. V. Nguyen, M.S. Thesis, Texas A\&M University, College Station, TX (1985).

14. J. S. Newman, "Electrochemical Systems," PrenticeHall, Englewood Cliffs, NJ (1973).

15. D. J. Pickett, "Electrochemical Reactor Design," 2nd ed., Elsevier Scientific Publishing, New York (1979). 